Review

\title{
Molecular Staging of Node Negative Patients with Colorectal Cancer
}

\author{
Terry Hyslop and Scott A. Waldman ${ }^{\bowtie}$ \\ Department of Pharmacology and Experimental Therapeutics, Thomas Jefferson University, Philadelphia, Pennsylvania, USA \\ Corresponding author: Scott A. Waldman, MD, PhD, 132 South 10 th Street, 1170 Main, Philadelphia, PA 19107, USA. \\ scott.waldman@jefferson.edu \\ (C) Ivyspring International Publisher. This is an open-access article distributed under the terms of the Creative Commons License (http://creativecommons.org/ \\ licenses/by-nc-nd/3.0/). Reproduction is permitted for personal, noncommercial use, provided that the article is in whole, unmodified, and properly cited.
}

Received: 2013.0I.10; Accepted: 2013.02.07; Published: 2013.03.0I

\begin{abstract}
Metastatic disease is the principle cause of death from colorectal cancer. In that context, the most significant indicator of overall survival and therapeutic response to adjuvant chemotherapy is the presence of metastatic tumor cells in regional lymph nodes. Although histopathologic analysis of lymph nodes is central to all colorectal cancer staging paradigms, its prognostic and predictive value is limited. Indeed, about $30 \%$ of patients with histopathology-negative lymph nodes (pNO) die from metastatic disease, reflected by microscopic lymph node metastases that are overlooked by standard techniques. These unrecognized tumor cells are especially important when considering racial disparities in outcomes in colorectal cancer patients, where blacks with lymph node-negative disease have the largest discrepancies in outcomes, with more than $40 \%$ excess mortality compared to Caucasian patients. However, the significance of tumor cells in regional lymph nodes remains uncertain, and approximately $50 \%$ of colorectal cancer patients with nodal metastases detected by histopathology remain free of recurrent disease. Accurate identification of occult metastases in regional lymph nodes, and defining their value as prognostic markers of recurrence risk and predictive markers of response to adjuvant chemotherapy remains one challenge in the management of colorectal cancer patients. Guanylyl cyclase C (GUCY2C), a receptor which is expressed primarily in intestinal cells normally, but is universally over-expressed by colorectal cancer cells, has been validated to detect prognostically significant occult metastases using quantitative RT-PCR (RT-qPCR). Biomarker validation was achieved through a prospective, multicenter, blinded clinical trial. In that trial, occult tumor burden estimated across all regional lymph nodes by GUCY2C RT-qPCR predicted clinical outcomes, identifying node-negative patients with a low (near zero) risk, and those with $>80 \%$ risk, of developing disease recurrence. Moreover, there was disproportionately higher occult tumor burden in black, compared to white, patients which contributes to racial disparities in outcomes in colorectal cancer. The diagnostic paradigm quantifying occult tumor burden using GUCY2C qRT-PCR is positioned to reduce racial disparities in colorectal cancer mortality.
\end{abstract}

Key words: Colorectal cancer; Early detection; Molecular stage; Cancer biomarkers; Guanylyl cyclase C

\section{Introduction}

Metastases continue to be the principle cause of mortality from colorectal cancer. In colorectal cancer staging patients, the most significant prognostic indicator of survival and predictive indicator of re- sponse to adjuvant therapy are metastatic cancer cells in regional lymph nodes [1-3]. While histopathologic assessment of lymph nodes is a core element of colorectal cancer staging algorithms, the prognostic and 
predictive value of lymph node metastases is restricted [1-5]. Thus, about 30\% of patients with histopathology-negative regional lymph nodes (stage I and II) develop recurrent disease, likely reflecting the presence of undetected metastases in those lymph nodes [1-3]. Moreover, these undetected metastases take on particular significance when considering racial disparities in colorectal cancer outcomes: African Americans with node-negative colorectal cancer are disadvantaged, with more than $40 \%$ excess mortality compared to Caucasian patients with the same disease [6-9]. However, the absolute significance of metastatic tumor cells in lymph nodes remains unclear, and approximately $50 \%$ of patients with lymph node metastases identified by histopathology (stage III) remain free of disease recurrence [1-3]. This discussion highlights the central importance of understanding how to identify regional lymph node metastases and evaluate their prognostic and predictive significance in managing black and white patients with colorectal cancer $[1-5,10]$.

Guanylyl cyclase C (GUCY2C) is a receptor which is typically restricted to intestinal epithelial cells normally, but is universally over-expressed by colorectal cancer cells. Because of this unique expression pattern, GUCY2C was validated to detect prognostically significant occult colorectal metastases by quantitative RT-PCR (RT-qPCR). Validation was achieved through a prospective, multicenter, blinded clinical trial [11]. This biomarker platform has been employed to generate a molecular staging algorithm that quantifies occult metastatic cancer burden across the regional lymph node network. In turn, this approach identifies histopathology node-negative colorectal cancer patients with near-zero risk, and those with $>80 \%$ risk, of developing disease recurrence [12-14]. Moreover, there is disproportionate occult tumor burden in African American, compared to Caucasian, colorectal cancer patients which contributes to racial disparities in outcomes in this disease [12].

\section{Colorectal Cancer}

Colorectal cancer continues to be the fourth most common cause of cancer, with about 140,000 new cases annually, and the second leading cause of cancer mortality, resulting in approximately $10 \%$ of cancer-related deaths, or $\sim 50,000$ patients annually, in the U.S. $[1,3,15]$. The significant impact of this disease can best be appreciated by considering the communities at risk, which in the U.S. include $>100$ million people $>50$ years. In colorectal cancer, mortality reflects metastatic disease, and about $20 \%$ of patients have unresectable disease at presentation while more than $30 \%$ develop metastases after diagnosis. Surgery continues to be the mainstay of management [1-3]. Yet, while "curative" surgery removes obvious tumor, occult metastatic disease produces recurrences [1-3]. Indeed, recurrence rates range from about $10 \%$ for disease limited to the epithelium (stage I) to more than $60 \%$ for cancer that has metastasized to more than 4 regional lymph nodes (stage III) [1-3].

\section{Staging}

The most significant prognostic marker of risk of disease recurrence in colorectal cancer is metastatic tumor cells in regional lymph nodes [1-3]. However, current approaches to detecting metastases in regional nodes remain inaccurate and their clinical significance is not certain. While histopathology is the standard approach, imprecision in staging by standard light microscopy signifies significant methodological limitations $[1-5,11]$. Thus, this approach is insensitive, with the limit for detection of $\sim 1$ cancer cell in 200 normal cells in lymph nodes [16]. Further, typically only one or a few sections from each lymph node are examined, leaving more than $99.99 \%$ of each specimen not reviewed, producing sampling error. Such limitations are evident when post-resection disease recurrence frequency is considered. Stage I and II disease, limited to the intestinal wall without obvious evidence of spread beyond the bowel, should be curable by surgery. Yet, rates of recurrence can be as high as $15 \%$ in stage I and $30 \%$ in stage II disease [1-3, 17]. Conversely, while all stage III patients have metastases in regional lymph nodes, only about $50 \%$ develop recurrent metastatic disease. Thus, about $50 \%$ of colorectal patients with nodal metastases visible by microscopy remain disease-free. In that context, molecular markers that certify the clinical significance of metastases in regional lymph nodes have yet to be identified. Indeed, identifying regional lymph node metastases and their clinical importance represents an important gap in managing colorectal cancer patients [10].

Beyond predicting disease recurrence, metastases in regional lymph nodes identify patients who benefit from adjuvant chemotherapy. Adjuvant chemotherapy incorporating 5-fluorouracil (5-FU) and irinotecan or oxaliplatin, increases 5 year survival from $\sim 40 \%$ (untreated) to $\sim 50 \%$ (treated) in patients with stage III colon cancer $[2,18]$. Limited therapeutic benefit in some, but not all, patients with stage III disease probably reflects stage heterogeneity, in which some patients have clinically aggressive metastases. One limitation to managing these patients is the inability to recognize clinically significant regional lymph node metastases, resulting in toxic therapies 
being administered to patients who derive no benefit, and may suffer adverse reactions. This scenario is even more complex for patients with stage I and II disease who are lymph node-negative by histopathology. Indeed, here, the utility of therapy is uncertain, with small survival benefits revealed for stage II patients in only some clinical trials $[18,19]$. This discussion highlights the unmet need for approaches that detect occult tumor cells and define their prognostic and predictive value, to identify colorectal cancer patients who derive the greatest benefit from therapy [10].

\section{Racial disparities}

While mortality from colorectal cancer decreases, there is an increasing racial gap in incidence and outcomes $[9,20,21]$. There is about a $20 \%$ greater incidence of, and approximately a $40 \%$ greater death rate from, colorectal cancer in African American, compared to Caucasian, patients $[7,9,20,21]$. These disparities reflect advanced stage at diagnosis, socioeconomic factors, and differences in disease management [6-9, 22-24]. However, beyond these parameters which influence overall disease outcomes, there are racial differences in stage-specific outcomes $[6,8$, $9,25]$. Unexpectedly, the biggest differences in outcomes occur in disease at the earliest stages, with more than $40 \%$ excess mortality in African Americans with lymph node-negative colorectal cancer, compared to Caucasians. These stage-specific differences do not reflect socioeconomic factors, healthcare access, or customs $[6,8,9,25]$. However, they could reflect greater occult metastases in regional lymph nodes $[8,9]$. Molecular approaches, which can quantify small nests of metastatic tumor cells, represent a technological opportunity to reduce racial disparities in colorectal cancer mortality [5, 11, 26-29].

\section{Enabling technologies}

Histopathology underestimates regional lymph node metastases, reflecting limitations in tissue volumes sampled and detection sensitivity (one tumor cell in 200 normal cells) [16]. The significance of these restrictions can be understood by considering clinically-impactful categories of lymph node metastases, including those that are greater than $0.2 \mathrm{~cm}$ (significant), micrometastases between 0.02 to $0.2 \mathrm{~cm}$ (indeterminate significance), and isolated tumor cells less than $0.02 \mathrm{~cm}$ (low significance) [1, 3]. In that context, evolving molecular approaches provide highly sensitive techniques that detect and characterize small quantities of cancer cells at metastatic foci. Enabling technologies, including RT-qPCR, may offer the most sensitive and specific detection of metastases [4,5].
Molecular approaches to staging offer advantages that overcome limitations in histopathology, with the ability to sample the entire specimen, overcoming limitations in tissue sampling, and detect one tumor cell in 107 normal cells, overcoming limitations in sensitivity $[4,5]$. Early experience with RT-PCR has been heterogeneous, because of inadequate sample size, clinical follow-up, and techniques. However, meta-analyses reveal the prognostic utility of occult metastases in histopathology-negative regional lymph nodes detected by RT-PCR in colorectal cancer patients $[4,5]$.

\section{GUCY2C is a molecular marker in colo- rectal cancer}

GUCY2C, selectively expressed by intestinal epithelial cells, is the receptor for the paracrine factors guanylin and uroguanylin, whose binding to the extracellular domain activates the catalytic domain in the cytoplasm, producing cGMP accumulation [11, 30, 31]. GUCY2C regulates epithelial homeostasis, coordinating the cell cycle, chromosomal stability, metabolism, and microenvironmental interactions organizing the crypt-surface axis along the colon and rectum [32]. Guanylin and uroguanylin are gene products universally lost early in in colorectal tumorigensis in animals and humans. GUCY2C silencing in mice increases tumorigenesis, reflecting dysregulation of cell proliferation and accumulation of DNA damage. Thus, GUCY2C is a tumor suppressor coordinating epithelial homeostasis whose silencing through hormone loss contributes to tumorigenesis [32].

The unique expression pattern of GUCY2C, restricted to intestinal epithelial cells normally, suggested that this receptor might be a biomarker for colorectal cancer. Indeed, GUCY2C was expressed by $>1,000$ specimens of normal intestine, but not by $>1,000$ extra-gastrointestinal tissues [11, 30, 31]. Also, GUCY2C mRNA $(n>900)$ and protein $(n>200)$ were detected in almost all primary and metastatic human colorectal tumors, but not in extra-gastrointestinal tumors (>200). Moreover, GUCY2C mRNA and protein are over-expressed by nearly all colorectal tumors. Taken together, these observations underscore the utility of GUCY2C as a marker for staging patients with colorectal cancer.

\section{Clinical utility of GUCY2C RT-qPCR for staging}

The utility of GUCY2C RT-PCR as a categorical variable (yes/no) for detecting occult tumor cells in regional lymph nodes that predict outcomes [27, 33] was defined in a prospective multicenter blinded 
clinical trial. This trial was supported by an analytically validated sensitive, robust, and reproducible quantitative RT-PCR (RT-qPCR) method for GUCY2C $[34,35]$. Further, it required unique statistical paradigms for accurate quantification of GUCY2C concentrations that could stratify the prognostic risk of cohorts of patients providing different numbers of regional lymph nodes for analysis $[11,36]$. In this trial, 257 patients with lymph node-negative (stage I and II) colorectal cancer were prospectively enrolled at 9 hospitals, providing 2,570 lymph nodes for analysis by histopathology and RT-qPCR. Patients were tracked for a median of 24 months and main outcomes were time to recurrence and disease-free survival [11]. Thirty-two $(12.5 \%)$ patients had nodes that were negative for GUCY2C, and thirty remained free of disease (recurrence rate 6.3\% [95\% CI 0.8-20.8\%]). Conversely, $225(87.5 \%)$ patients had regional lymph nodes that were positive for GUCY2C, and $47 \quad(20.9 \%$ [15.8-26.8\%]) developed recurrent disease $(\mathrm{P}=0.006)$. Indeed, GUCY2C in regional lymph nodes was the most powerful independent marker of prognosis, and patients who were GUYC2C-positive had earlier time to recurrence (adjusted hazard ratio 4.66 [1.11-19.57]; $\mathrm{P}=0.035$ ) and lower disease-free survival (adjusted hazard ratio 3.27 [1.15-9.29]; $\mathrm{P}=0.026$ ). Of significance, this is one of the first prospective multicenter blinded clinical trial offering level I evidence associating molecular lymph node metastases detected by RT-PCR with disease recurrence [11]. This trial reveals the utility of GUCY2C RT-qPCR to detect occult metastatic tumor cells that define disease outcomes. This approach is quite robust with independent validation by other laboratories, operators and technology platforms [37-39].

Although a high proportion of histologically node-negative patients harbor molecular metastases by GUCY2C RT-qPCR, most of these patients will never recur [1-3]. Understanding this apparent inconsistency depends on the realization that the presence of regional lymph node metastases does not insure disease recurrence but, rather, indicates risk of disease recurrence. This inability to identify clinically significant from insignificant lymph node metastases represents one key gap in the management of patients with colorectal cancer. Indeed, this uncertainty can be appreciated by considering that only about $50 \%$ of stage III patients develop recurrent disease although all have metastases in regional lymph nodes detectable by histopathology [1-3].

Here, the uncertain clinical significance of occult metastases underscores the limitations of qualitative RT-PCR generally for categorical identification of occult metastases, which is the absence of quantitative information about metastatic burden [5]. The enhanced sensitivity of RT-PCR, with its maximized tissue sampling and ability to discriminate single cells, reveals metastases below the limit of prognostic risk $[1,5,11,28]$, restricting the specificity of this approach. There is an evolving approach that extends beyond the categorical detection of cancer cells, to quantify occult metastatic burden (how much) across the regional lymph node web to define disease risk and clinical outcomes. This algorithm originates in, and extends, established histopathological concepts. First, a quantitative relationship exists between prognostic risk and the number of nodes containing metastatic cancer cells by histopathology. For example, stage III patients with more than 4 involved regional lymph nodes exhibit a recurrence rate which is greater than patients with fewer than 3 involved lymph nodes [1-3]. Moreover, a quantitative relationship exists between the volume of metastatic tumor cells in each lymph node and prognostic risk. In that context, metastases greater than $0.2 \mathrm{~cm}$ are associated with increased cancer recurrence while the relationship between individual tumor cells or nests less than $0.02 \mathrm{~cm}$ and risk is unclear [1-3]. The development of quantitative RT-PCR (RT-qPCR) offers a remarkable opportunity to quantify occult metastases to define prognostic risk and predict the benefit of adjuvant chemotherapy. Indeed, quantification of GUYC2C expression offers a molecular homologue of morphological analyses of metastatic volumes in regional lymph nodes. Here, quantification by RT-qPCR enhances 2-dimensional morphology by estimating the amount of metastases in large volumes of lymph node materials, rather than thin sections, and across all lymph nodes to estimate occult metastatic burden throughout the regional lymph node web.

Thus, we designed analytic approaches to define the association of occult metastatic burden, quantified by GUCY2C RT-qPCR, with outcomes in colorectal cancer patients to examine the quantitative relationship between occult nodal metastases and prognostic risk $[11,13]$. In this paradigm, relationships between clinical outcomes, including time to recurrence and disease-free survival, and occult metastatic burden, were estimated by recursive partitioning [13]. Here, $176(60 \%)$ stage I-II colon cancer patients exhibited low tumor burden (MolLow), and all but 4 remained free of disease (recurrence rate 2.3\% [95\% CI 0.1-4.5\%]). Further, 90 (31\%) colon cancer patients exhibited intermediate tumor burden (MolInt) and 30 (33.3\% [23.7\%-44.1\%]) developed disease recurrence. Moreover, $25(9 \%)$ colon cancer patients had high tumor burden (MolHigh), and $17 \quad(68.0 \%$ [46.5\%-85.1\%] developed recurrent disease $(\mathrm{P}<0.001)$. 
Occult tumor burden was an independent marker of prognosis and patients with MolInt and MolHigh disease exhibited a graded risk of earlier time to recurrence (MolInt, adjusted hazard ratio 25.52 [11.08-143.18]; $\mathrm{P}<0.001 ;$ MolHigh, 65.38 [39.01-676.94]; $\mathrm{P}<0.001$ ) and lower disease-free survival (MolInt, 9.77 [6.26-87.26]; $\mathrm{p}<0.001 ;$ MolHigh, 22.97 [21.59-316.16]; $\mathrm{P}<0.001)$. This paradigm offers a remarkable enhancement to the use of GUCY2C as a categorical marker, where $88 \%$ of colorectal cancer patients were GUCY2C-positive with a recurrence risk of 20\% [11]. They underscore the clinical opportunity offered by occult metastatic burden analysis to assign prognostic risk to patients with lymph node-negative colorectal cancer. In that context, identifying patients with a mortality risk equal to patients with disseminated metastases underscores the prognostic value of occult metastatic burden analysis. Additionally, patients with the greatest occult metastatic burden might benefit most from receiving adjuvant chemotherapy.

\section{Racial disparities reflect differences in occult metastatic burden}

Prospective analysis of GUCY2C to detect occult metastases in regional lymph nodes $[11,13]$ provided an opportunity to define the contribution of occult metastatic burden to racial disparities in outcomes in colon cancer [12]. Indeed, there was 4 -fold greater levels of occult metastases in individual nodes in 23 African American, compared to 259 Caucasian, patients with colon cancer ( $p<0.001 ; 95 \% \mathrm{CI}=3.3,6.7)$. Occult metastatic burden across the regional lymph node web stratified the whole population into categories with low $(60 \%$; recurrence rate $(R R)=2.3 \%[95 \%$ CI $0.1-4.5 \%])$, intermediate $(31 \%$; $R R=33.3 \%$ $[23.7 \%-44.1 \%])$, and high (9\%; $\quad \mathrm{RR}=68.0 \%$ $[46.5 \%-85.1 \%], \mathrm{P}<0.001)$ risk. However, race $(\mathrm{P}=0.02)$, $\mathrm{T}$ stage $(\mathrm{p}=0.02)$, and number of lymph nodes collected for histology $(\mathrm{P}=0.003)$ were independent prognostic markers of risk. African American patients, compared to Caucasian patients, were most likely to have levels of occult metastatic burden producing the greatest risk (adjusted odds ratio $=5.08$ $[1.55,16.65] ; P=0.007)$. These observations underscore occult metastatic burden as one determinant contributing to racial disparities in stage-specific outcomes in colorectal cancer. As a correlate, they suggest that occult metastatic burden may represent a detect-treat algorithm to reduce racial disparities in mortality in colorectal cancer.

\section{Number of lymph nodes optimizes the accuracy of occult metastatic analysis}

Not surprisingly, the accuracy of estimating oc- cult metastatic burden is dependent on the number of regional lymph nodes analyzed by RT-qPCR [14]. Indeed, patients providing fewer than 5 regional lymph nodes exhibited occult metastatic burdens that identified more than $95 \%$ of stage I and II patients as low and intermediate risk, with few patients in the highest risk category. Conversely, analysis of more than 12 regional lymph nodes optimally resolved the lowest risk cohort, representing $68 \%$ of stage I and II patients. Further, analysis of more than 23 regional lymph nodes entirely eliminated the intermediate risk cohort, maximizing the identification of patients with the greatest recurrence risk, representing approximately $28 \%$ of stage I and II patients. Thus, the prognostic utility of occult metastatic burden is related to the number of regional lymph nodes analyzed by RT-qPCR. Indeed, these data suggest that the intermediate risk category reflects inaccuracies in estimating occult tumor burden due to insufficient nodal collections. Based on these data, analysis of more than 12 nodes offers estimates of occult metastatic burden that maximally define clinical outcomes, resolving stage I and II cohorts into patients with (1) with a near-zero likelihood of recurrence $(\sim 70 \%)$ and (2) a maximum likelihood (>70\%) of recurrence $(30 \%)$ [14]. Indeed, it may be the case that this algorithm provides near-perfect prognostic risk stratification, since $\sim 70 \%$ of node-negative patients are cured by surgery, while $\sim 30 \%$ develop disease recurrence [1-3].

\section{Future perspectives}

Staging patients with colorectal cancer involves histopathological analyses of tumors and regional lymph nodes. However, this paradigm under-estimates the presence of metastases, and about $30 \%$ of node-negative patients progress to developing disease recurrence. Limitations in established histopathology techniques, including tissue sampling and sensitivity, can be overcome using emerging molecular approaches. Indeed, identification of occult metastases in regional lymph nodes is the most powerful independent indicator of prognostic risk in colorectal cancer patients. Prospective clinical assessment indicates that analyses of lymph nodes using RT-qPCR estimates metastatic burden that identifies those stage I and II patients that will develop recurrent disease. Further, these approaches suggest that occult metastatic burden is one contributor to racial disparities in outcomes in African American, compared to Caucasian, patients with colorectal cancer. Moreover, these at-risk stage I and II patients, black and white, may remarkably benefit from adjuvant chemotherapy.

Beyond lymph node analysis, genomic paradigms are evolving to extract clinically-important 
information from tumors to enhance staging algorithms to improve outcomes. Mutations in oncogenes or tumor suppressors, transcriptomic analyses, proteomic and metabolomic signatures, and epigenetic profiling in tumors can personalize evaluations of risk, identify patients who could benefit from treatment, and predict which treatments will be clinically active [40-44]. However, the clinical value of molecular analyses of primary tumors is only relevant in the context of whether primary tumors have metastasized. Indeed, tumors with molecular signature suggesting a poor prognosis represent a risk only if they are not excised before metastasizing. In that context, molecular approaches profiling tumors might be of greatest utility when applied to patients with occult metastatic burden, rather than to those free of metastases in regional lymph nodes. Here, molecular analyses of lymph nodes provides an opportunity to prioritize expensive tumor analyses to optimize cost-effective healthcare [11]. Future clinical studies will explore the utility of analytical algorithms where patients who are node-negative by histopathology are reflexed to molecular staging, to quantify occult metastatic burden, followed subsequently by molecular tumor profiling for patients at increased prognostic risk, to define treatments individualized to their tumor biology [45].

As a last note, while the technology for RT-qPCR is evolving, it remains primarily the province of specialty laboratories, with only modest penetration into hospitals and medical centers. However, molecular diagnostics is growing business, in excess of $\$ 14$ billion with projected growth of $10 \%$ annually $[46,47]$. In that context, molecular diagnostics approved by the FDA expanded from 72 in 2006 to 134 in 2009 [48]. Further, molecular tests developed in specialty laboratories were in excess of 1,400 in 2009 [49]. These data suggest that molecular diagnostics will be increasingly integrated into the clinical care of patients. While specialty laboratories provide the experience and validated analytic algorithms consistent with FDA guidelines and CMS reimbursement in the short term, these enabling platforms will support integration of molecular staging into disease management algorithms across the entire healthcare enterprise in the long term.

\section{Acknowledgements}

This work was supported by funding from the National Institutes of Health (CA75123, CA95026, CA112147, CA146033, CA170533), Targeted Diagnostic \& Therapeutics, Inc., and the Pennsylvania Department of Health (SAP \#4100059197, SAP \#4100051723). The Pennsylvania Department of
Health specifically disclaims responsibility for any analyses, interpretations or conclusions. SAW is the Samuel M.V. Hamilton Endowed Professor.

\section{Competing Interests}

SAW is the Chair (uncompensated) of the Scientific Advisory Board of Targeted Diagnostics and Therapeutics, Inc., which provided research funding that, in part, supported this study and which has a license to commercialize inventions related to this work, and the Chair of the Data Safety and Monitoring Committee for the C-Cure Trial sponsored by Cardio3 (Belgium).

\section{References}

1. Compton CC, Greene FL. The staging of colorectal cancer: 2004 and beyond. CA Cancer J Clin. 2004; 54: 295-308. doi:54/6/295 [pii].

2. Cunningham D, Atkin W, Lenz HJ, Lynch HT, Minsky B, Nordlinger B, et al. Colorectal cancer. Lancet. 2010; 375: 1030-47.

3. Edge SB, Byrd DR, Compton CC, Fritz AG, Greene FL, Trotti A. AJCC Cancer Staging Manual. 7th ed. New York: Springer; 2009.

4. Iddings D, Ahmad A, Elashoff D, Bilchik A. The prognostic effect of micrometastases in previously staged lymph node negative (N0) colorectal carcinoma: a meta-analysis. Ann Surg Oncol. 2006; 13: 1386-92. doi:10.1245/s10434-006-9120-y.

5. Nicastri DG, Doucette JT, Godfrey TE, Hughes SJ. Is occult lymph node disease in colorectal cancer patients clinically significant? A review of the relevant literature. J Mol Diagn. 2007; 9: 563-71. doi:jmoldx.2007.070032 [pii]10.2353/jmoldx.2007.070032.

6. Chien C, Morimoto LM, Tom J, Li CI. Differences in colorectal carcinoma stage and survival by race and ethnicity. Cancer. 2005; 104: 629-39. doi:10.1002/cncr.21204.

7. Le H, Ziogas A, Lipkin SM, Zell JA. Effects of socioeconomic status and treatment disparities in colorectal cancer survival. Cancer Epidemiol Biomarkers Prev. 2008; 17: 1950-62. doi:17/8/1950 [pii]10.1158/1055-9965.EPI-07-2774.

8. Marcella S, Miller JE. Racial differences in colorectal cancer mortality. The importance of stage and socioeconomic status. J Clin Epidemiol. 2001; 54: 359-66. doi:S0895-4356(00)00316-4 [pii].

9. Soneji S, Iyer SS, Armstrong K, Asch DA. Racial disparities in stage-specific colorectal cancer mortality: 1960-2005. Am J Public Health. 2010; 100: 1912-6.

10. Grothey A. Does stage II colorectal cancer need to be redefined? Clin Cancer Res. 2011; 17: 3053-5.

11. Waldman SA, Hyslop T, Schulz S, Barkun A, Nielsen K, Haaf J, et al. Association of GUCY2C expression in lymph nodes with time to recurrence and disease-free survival in pN0 colorectal cancer. JAMA. 2009; 301: 745-52. doi:301/7/745 [pii]10.1001/jama.2009.141.

12. Hyslop T, Weinberg DS, Schulz S, Barkun A, Waldman SA. Occult tumor burden contributes to racial disparities in stage-specific colorectal cancer outcomes. Cancer. 2012;118(9):2532-40.

13. Hyslop T, Weinberg DS, Schulz S, Barkun A, Waldman SA. Occult tumor burden predicts disease recurrence in lymph node-negative colorectal cancer. Clin Cancer Res. 2011; 17: 3293-303.

14. Hyslop T, Weinberg DS, Schulz S, Barkun A, Waldman SA. Analytic lymph node number establishes staging accuracy by occult tumor burden in colorectal cancer. J Surg Oncol. 2012; 106: 24-30.

15. Siegel R, Ward E, Brawley O, Jemal A. Cancer statistics, 2011: The impact of eliminating socioeconomic and racial disparities on premature cancer deaths. CA Cancer J Clin. 2011;61(4):212-36.

16. Ratto C, Sofo L, Ippoliti M, Merico M, Bossola M, Vecchio FM, et al. Accurate lymph-node detection in colorectal specimens resected for cancer is of prognostic significance. Dis Colon Rectum. 1999; 42: 143-54.

17. Radel C, Martus P, Papadoupolos T, Fazesi L, Klimpfinger M, Fietkau R, et al. Prognostic significance of tumor regression after preoperative chemoradiotherapy for rectal cancer. Journal of Clinical Oncology. 2005; 23: 8688-96. doi:10.1200/jco.2005.02.1329.

18. Meyerhardt JA, Mayer RJ. Systemic therapy for colorectal cancer. N Engl J Med. 2005; 352: 476-87. doi:352/5/476 [pii]10.1056/NEJMra040958. 
19. Benson AB, 3rd, Schrag D, Somerfield MR, Cohen AM, Figueredo AT, Flynn PJ, et al. American Society of Clinical Oncology recommendations on adjuvant chemotherapy for stage II colon cancer. J Clin Oncol. 2004; 22: 3408-19. doi:10.1200/JCO.2004.05.063JCO.2004.05.063 [pii].

20. Polite BN, Dignam JJ, Olopade OI. Colorectal cancer and race: understanding the differences in outcomes between African Americans and whites. Med Clin North Am. 2005; 89: 771-93. doi:S0025-7125(05)00024-6 [pii]10.1016/j.mcna.2005.03.001.

21. Polite BN, Dignam JJ, Olopade OI. Colorectal cancer model of health disparities: understanding mortality differences in minority populations. J Clin Oncol. 2006; 24: 2179-87.

22. Laiyemo AO, Doubeni C, Pinsky PF, Doria-Rose VP, Bresalier R, Lamerato LE, et al. Race and colorectal cancer disparities: health-care utilization vs different cancer susceptibilities. J Natl Cancer Inst. 2010; 102: $538-46$.

23. Mayberry RM, Coates RJ, Hill HA, Click LA, Chen VW, Austin DF, et al. Determinants of black/white differences in colon cancer survival. J Natl Cancer Inst. 1995; 87: 1686-93.

24. White A, Vernon SW, Franzini L, Du XL. Racial disparities in colorectal cancer survival: to what extent are racial disparities explained by differences in treatment, tumor characteristics, or hospital characteristics? Cancer. 2010; 116: 4622-31.

25. Chen VW, Fenoglio-Preiser CM, Wu XC, Coates RJ, Reynolds P, Wickerham DL, et al. Aggressiveness of colon carcinoma in blacks and whites. National Cancer Institute Black/White Cancer Survival Study Group. Cancer Epidemiol Biomarkers Prev. 1997; 6: 1087-93.

26. Bilchik AJ, Hoon DS, Saha S, Turner RR, Wiese D, DiNome M, et al. Prognostic impact of micrometastases in colon cancer: interim results of a prospective multicenter trial. Ann Surg. 2007; 246: 568-75.

27. Cagir B, Gelmann A, Park J, Fava T, Tankelevitch A, Bittner EW, et al. Guanylyl cyclase $\mathrm{C}$ messenger RNA is a biomarker for recurrent stage II colorectal cancer. Ann Intern Med. 1999; 131: 805-12.

28. Iddings D, Bilchik A. The biologic significance of micrometastatic disease and sentinel lymph node technology on colorectal cancer. J Surg Oncol. 2007; 96: 671-7. doi:10.1002/jso.20918.

29. Liefers GJ, Cleton-Jansen AM, van de Velde CJ, Hermans J, van Krieken $\mathrm{JH}$, Cornelisse CJ, et al. Micrometastases and survival in stage II colorectal cancer. N Engl J Med. 1998; 339: 223-8.

30. Carrithers SL, Barber MT, Biswas S, Parkinson SJ, Park PK, Goldstein SD, et al. Guanylyl cyclase C is a selective marker for metastatic colorectal tumors in human extraintestinal tissues. Proc Natl Acad Sci U S A. 1996; 93: 14827-32.

31. Frick GS, Pitari GM, Weinberg DS, Hyslop T, Schulz S, Waldman SA. Guanylyl cyclase C: a molecular marker for staging and postoperative surveillance of patients with colorectal cancer. Expert Rev Mol Diagn. 2005; 5: 701-13.

32. Pitari GM, Li P, Lin JE, Zuzga D, Gibbons AV, Snook AE, et al. The paracrine hormone hypothesis of colorectal cancer. Clin Pharmacol Ther. 2007; 82: 441-7.

33. Waldman SA, Cagir B, Rakinic J, Fry RD, Goldstein SD, Isenberg G, et al. Use of guanylyl cyclase $\mathrm{C}$ for detecting micrometastases in lymph nodes of patients with colon cancer. Dis Colon Rectum. 1998; 41: 310-5.

34. Chervoneva I, Hyslop T, Iglewicz B, Johns L, Wolfe HR, Schulz S, et al. Statistical algorithm for assuring similar efficiency in standards and samples for absolute quantification by real-time reverse transcription polymerase chain reaction. Anal Biochem. 2006; 348: 198-208. doi:S0003-2697(05)00776-1 [pii]10.1016/j.ab.2005.10.042.

35. Schulz S, Hyslop T, Haaf J, Bonaccorso C, Nielsen K, Witek ME, et al. A validated quantitative assay to detect occult micrometastases by reverse transcriptase-polymerase chain reaction of guanylyl cyclase $\mathrm{C}$ in patients with colorectal cancer. Clin Cancer Res. 2006; 12: 4545-52.

36. Chervoneva I, Li Y, Iglewicz B, Waldman S, Hyslop T. Relative quantification based on logistic models for individual polymerase chain reactions. Stat Med. 2007; 26: 5596-611. doi:10.1002/sim.3127.

37. Beaulieu M, Desaulniers M, Bertrand N, Deschesnes RG, Beaudry G, Garon $\mathrm{G}$, et al. Analytical performance of a qRT-PCR assay to detect guanylyl cyclase $\mathrm{C}$ in FFPE lymph nodes of patients with colon cancer. Diagn Mol Pathol. 2010; 19: 20-7.

38. Haince JF, Houde M, Beaudry G, L'Esperance S, Garon G, Desaulniers $\mathrm{M}$, et al. Comparison of histopathology and RT-qPCR amplification of guanylyl cyclase $\mathrm{C}$ for detection of colon cancer metastases in lymph nodes. J Clin Pathol. 2010; 63: 530-7.

39. Sargent DJ, Resnick MB, Meyers MO, Goldar-Najafi A, Clancy T, Gill S, et al. Evaluation of Guanylyl Cyclase C Lymph Node Status for Colon Cancer Staging and Prognosis. Ann Surg Oncol. 2011 Nov;18(12):3261-70.
40. Croner RS, Peters A, Brueckl WM, Matzel KE, Klein-Hitpass L, Brabletz $\mathrm{T}$, et al. Microarray versus conventional prediction of lymph node metastasis in colorectal carcinoma. Cancer. 2005; 104: 395-404. doi:10.1002/cncr.21170

41. Frigola J, Song J, Stirzaker C, Hinshelwood RA, Peinado MA, Clark SJ. Epigenetic remodeling in colorectal cancer results in coordinate gene suppression across an entire chromosome band. Nat Genet. 2006; 38: 540-9. doi:ng1781 [pii]10.1038/ng1781.

42. Jen J, Kim H, Piantadosi S, Liu ZF, Levitt RC, Sistonen P, et al. Allelic loss of chromosome 18q and prognosis in colorectal cancer. N Engl J Med. 1994; 331: 213-21.

43. Paik S, Shak S, Tang G, Kim C, Baker J, Cronin M, et al. A multigene assay to predict recurrence of tamoxifen-treated, node-negative breast cancer. N Engl J Med. 2004; 351: 2817-26. doi:NEJMoa041588 [pii]10.1056/NEJMoa041588.

44. Wang Y, Jatkoe T, Zhang Y, Mutch MG, Talantov D, Jiang J, et al. Gene expression profiles and molecular markers to predict recurrence of Dukes' B colon cancer. J Clin Oncol. 2004; 22: 1564-71. doi:10.1200/JCO.2004.08.186JCO.2004.08.186 [pii].

45. Allegra CJ, Jessup JM, Somerfield MR, Hamilton SR, Hammond EH, Hayes DF, et al. American Society of Clinical Oncology provisional clinical opinion: Testing for KRAS gene mutations in patients with metastatic colorectal carcinoma to predict response to anti-epidermal growth factor receptor monoclonal antibody therapy. J Clin Oncol. 2009; 27: 2091-6. doi:10.1200/jco.2009.21.9170.

46. Wilson C, Schulz S, Waldman S. Cancer biomarkers: Where medicine, business, and public policy intersect. Biotechnology Healthcare. 2007.

47. Wilson C, Schulz S, Waldman SA. Biomarker development, commercialization, and regulation: individualization of medicine lost in translation. Clin Pharmacol Ther. 2007; 81: 153-5. doi:6100088 [pii]10.1038/sj.clpt.6100088

48. Holland C. FDA-cleared/approved molecular diagnostic tests. Association for Molecular Pathology; 2006.

49. Sun F, Breuning W, Uhl S, Ballard R, Tipton K, Schoelles K. Quality, regulation and clinical utility of laboratory-developed tests. Technology Assessment Report: AHRQ; 2009. 